\title{
Efficacy of levetiracetam in epilepsy and effect of concomitant drugs on the incidence of somnolence as an adverse effect
}

\author{
Hideki Shimomura $^{1}$, Kyoko Minagawa $^{1}$, Junji Mine ${ }^{1}$, Tomoko Lee ${ }^{1}$, Chikako Mure ${ }^{1}$, \\ Naoko Taniguchi ${ }^{1}$, Sachi Tokunaga ${ }^{1}$, Yasuhiko Tanaka ${ }^{1}$, Masumi Okuda ${ }^{1}$, Shogo Kikuchi ${ }^{2}$, \\ Yasuhiro Takeshima ${ }^{1}$ \\ ${ }^{1}$ Department of Pediatrics, Hyogo College of Medicine, 1-1 Mukogawa, Nishinomiya, Hyogo \\ 663-8501, Japan \\ 2 Department of Public Health, Aichi Medical University School of Medicine, 1-1 Yazakokari- \\ mata, Nagakute, Aichi 480-1195, Japan
}

Key words: Epilepsy treatment; Somnolence; Levetiracetam; Concomitant antiepileptic drugs

Received: February 15, 2021; Accepted: August 6, 2021

\section{Abstract}

Purpose: Somnolence is a major adverse effect associated with levetiracetam (LEV) administration in epilepsy treatment, and it can prompt patients to discontinue the drug. This study aimed to assess the efficacy of LEV and evaluate the incidence and risk factors of somnolence caused by LEV administration.

Methods: Medical records of patients with epilepsy treated with LEV between October 2010 and June 2018 were retrospectively analyzed to evaluate drug efficacy. We also analyzed the corresponding incidence of somnolence and its associated factors.

Results: Ninety-five patients were included in the study. Of these, 55 (58.9\%) showed more than $50 \%$ seizure reduction. There was no significant difference in efficacy between the monotherapy $(\mathrm{n}=38)$ and adjunctive therapy $(\mathrm{n}=57)$ groups. Twenty-nine patients $(30.5 \%)$ experienced somnolence, and nine patients (9.5\%) discontinued LEV. In total, nine patients $(23.7 \%)$ treated with LEV as monotherapy and 20 patients $(35.1 \%)$ treated with LEV as adjunctive therapy experienced somnolence, with no significant difference between the two groups. Regarding concomitant antiepileptic drug use, only valproic acid (VPA) significantly induced somnolence. Multiple logistic regression analysis showed that LEV as adjunctive therapy to VPA was a risk factor for somnolence (odds ratio, 3.95; 95\% confidence interval, 1.43-11.36; $\mathrm{p}=0.0079$ ).

Discussion: Our data showed that the efficacy of LEV and the incidence of somnolence due to LEV therapy were comparable to those reported previously. Incidence of somnolence increased when patients were treated with LEV as adjunctive therapy to VPA. Discontinuation of LEV due to somnolence might be decreased by avoiding adjunctive treatment with VPA. 


\section{Introduction}

Somnolence is one of the most common adverse effects associated with the use of antiepileptic drugs (AEDs). It can sometimes be more disabling than the seizures themselves. Therefore, it is important to choose appropriate AEDs to avoid adverse effects including somnolence.

Levetiracetam (LEV) is a modern antiepileptic drug with high efficacy and few drug interactions with other AEDs [1-3]. Moreover, it has been demonstrated to be as effective as conventional AEDs. Therefore, LEV has increasingly been preferred as a first-line AED in recent years. The two most common adverse effects of LEV are somnolence and behavioral problems. Somnolence reduces medication tolerability and affects further treatment, and $2.2-9.4 \%$ of patients are reportedly unable to continue LEV due to its adverse effects including somnolence [4, 5]. However, in the absence of somnolence, LEV consistently results in effective treatment.

Studies have reported that the incidence of adverse effects such as somnolence does not correlate with the number or dosage of concomitantly used AEDs [6, 7]. However, the correlation between each AED and adverse effects was not considered in these studies.

Therefore, we hypothesized that somnolence associated with LEV may develop due to its interaction with other AEDs. In the present study, we analyzed the efficacy of LEV, and elucidated the risk factors for developing somnolence due to LEV administration, especially as an adjunctive therapy to other AEDs.

\section{Methods}

Patients
We retrospectively analyzed the medical records of all patients treated with LEV in the pediatric department of Hyogo College of Medicine in Japan between October 2010 and June 2018. Data for age, sex, types of seizures, etiology, neurodevelopmental comorbidities, daily dose of LEV, concomitantly used AEDs (administered before LEV), seizure frequency, and adverse events were analyzed. We particularly focused on somnolence as an adverse effect in different modes of AED administration: LEV monotherapy and LEV therapy adjunctive to other AEDs. The need for informed consent from the patients was waived due to the retrospective nature of our study, and the study design was approved by the Review Board of Hyogo College of Medicine (approval number 3115). This study was conducted in accordance with the principles of Declaration of Helsinki and other nationally valid regulations and guidelines.

\section{Evaluations}

The types of seizures and epilepsy syndromes were classified based on the International League Against Epilepsy classification of epileptic seizures and epileptic syndromes [8]. The mean seizure frequency was calculated over a baseline period of at least 3 months prior to the initiation of LEV, and for an LEV treatment period of at least 3 months. Treatment responders were defined as those whose seizure frequency was reduced by at least $50 \%$ ( $50 \%$ responder rate).

Adverse effects were assessed based on the complaints extracted from the medical records that were spontaneously reported by the parents or the patients. In the medical rec- 
ords, somnolence was assessed based on increased sleep duration or increased frequency of sleep during the day.

\section{Statistical analysis}

JMP Pro 14 (SAS Institute Inc., Cary, NC, USA) was used in all statistical analyses. The effect of each variable and the incidence of somnolence were tested using Fisher's exact test. The relationship between daily LEV dose and treatment response or incidence of somnolence was tested using Mann-Whitney $\mathrm{U}$ test. Odds ratios (ORs) and 95\% confidence intervals (CIs) were calculated. The differences with $\mathrm{p}$ values less than 0.05 were considered statistically significant. A multiple logistic regression analysis was performed with somnolence as the outcome variable and adjunctive use of other drugs as exposure variables.

\section{Results}

Demographic data of the subjects included in this study are presented in Table 1. In total, 95 patients treated with LEV (45 males, 50 females; median age, 8 years; range, 3 months-26 years) were identified. Sixty-six patients $(69.5 \%)$ had focal onset seizures. The possible underlying etiologies of epilepsy were determined in $62(65.3 \%)$ patients, while no etiological factor was identified in $33(34.7 \%)$ patients. Thirty-nine patients (40\%) were bedridden due to underlying disease. Of the non-bedridden patients, 11 (11.6\%) had neurodevelopmental comorbidities including intellectual disabilities, autism spectrum disorders, and attention-deficit/ hyperactivity disorders. Fifty-seven patients $(60.0 \%)$ were administered adjunctive thera- py. The concomitantly used AEDs are listed in Table 1.

Data regarding the efficacy of LEV are shown in Table 2. Overall, $58.9 \%$ of all patients were responders. The $50 \%$ responder rate was the highest in patients administered

Table 1 Baseline clinical and demographic characteristics

\begin{tabular}{|c|c|}
\hline Patients enrolled, $n$ & 95 \\
\hline Age, median (range) years & $8(0-26)$ \\
\hline \multicolumn{2}{|l|}{ Sex } \\
\hline Male & $45(47.4)$ \\
\hline Female & $50(52.6)$ \\
\hline \multicolumn{2}{|l|}{ Type of seizure } \\
\hline Focal onset aware & $24(25.2)$ \\
\hline Focal onset impaired awareness & $30(31.6)$ \\
\hline Focal to bilateral tonic-clonic & $12(12.6)$ \\
\hline Generalized onset tonic-clonic & $7(7.4)$ \\
\hline Myoclonic & $13(13.7)$ \\
\hline Spasms & $5(5.3)$ \\
\hline Other (absence, atypical absence, atonic) & $3(3.2)$ \\
\hline Unknown & $1(1.1)$ \\
\hline \multicolumn{2}{|l|}{ Etiology of epilepsy } \\
\hline Structural & $44(46.3)$ \\
\hline Genetic & $14(14.7)$ \\
\hline Infectious & $3(3.2)$ \\
\hline Metabolic & $1(1.1)$ \\
\hline No etiologic factor & $33(34.7)$ \\
\hline Neurodevelopmental comorbidity & $11(11.6)$ \\
\hline \multicolumn{2}{|l|}{ Type of concomitantly used AEDs } \\
\hline None & $38(40.0)$ \\
\hline Valproic acid & $23(24.2)$ \\
\hline Lamotrigine & $21(22.1)$ \\
\hline Zonisamide & $12(12.6)$ \\
\hline Phenobarbital & $8(8.4)$ \\
\hline Clonazepam & $7(7.4)$ \\
\hline Topiramate & $6(6.3)$ \\
\hline Carbamazepine & $5(5.3)$ \\
\hline Other & $7(7.4)$ \\
\hline \multicolumn{2}{|l|}{ Number of concomitantly used AEDs } \\
\hline 0 (monotherapy) & $38(40.0)$ \\
\hline 1 & $29(30.5)$ \\
\hline 2 & $24(25.3)$ \\
\hline 3 & $4(4.2)$ \\
\hline
\end{tabular}

Data are presented as n (\%) unless specified otherwise. AEDs, antiepileptic drugs 
Table 2 Clinical variables related to the efficacy of levetiracetam.

\begin{tabular}{llll}
\hline Etiologic factor & Mode of therapy & Cases, $\mathrm{n}$ & $50 \%$ responder rate $(\%)$ \\
\hline Total & & 95 & 58.9 \\
Without an etiologic factor & Monotherapy & 20 & 70 \\
& Adjunctive therapy & 11 & 63.6 \\
\multirow{2}{*}{ With an etiologic factor } & Monotherapy & 18 & 61.1 \\
& Adjunctive therapy & 46 & 52.2 \\
\hline
\end{tabular}

monotherapy and for whom an etiologic factor $(70.0 \%)$ was not identified, and was lowest in patients with an identified etiologic factor who were administered adjunctive therapy (52.2\%). No significant difference in the efficacy of LEV was observed between the monotherapy and adjunctive therapy groups, irrespective of the presence or absence of underlying etiology. Furthermore, patients in the responder group received $10-80 \mathrm{mg} / \mathrm{kg}$ (median, $30 \mathrm{mg} / \mathrm{kg}$ ) of LEV daily and those in the non-responder group received 5-70 $\mathrm{mg} / \mathrm{kg}$ (median, $40 \mathrm{mg} / \mathrm{kg}$ ) of LEV daily; hence, the daily dose of LEV was not different between the two groups ( $p=0.20)$.

The incidence of somnolence as an adverse effect of LEV is shown in Table 3. Somnolence was reported by 29 patients $(30.5 \%)$, nine of whom $(9.5 \%)$ had to discontinue LEV therapy. In total, nine patients

Table 3 Incidence of somnolence.

\begin{tabular}{lll}
\hline Variables & Cases, n (\%) & p value* \\
\hline Total & $29 / 95(30.5)$ & \\
Monotherapy & $9 / 38(23.7)$ & Reference \\
Adjunctive therapy & $20 / 57(35.1)$ & 0.264 \\
& & \\
Concomitantly used AEDs & & \\
Valproic acid & $13 / 23(56.5)$ & 0.00473 \\
Lamotrigine & $6 / 21(28.6)$ & 1 \\
Zonisamide & $0 / 12(0)$ & 0.0958 \\
Phenobarbital & $1 / 8(12.5)$ & 0.4285 \\
Clonazepam & $3 / 7(42.9)$ & 0.6748 \\
Carbamazepine & $2 / 5(40.0)$ & 0.3215 \\
Topiramate & $0 / 6(0)$ & 0.172 \\
Other & $1 / 13(14.3)$ & - \\
\hline *Versus levetiracetam & monotherapy. AEDs, \\
antiepileptic drug. & &
\end{tabular}

(23.7\%) who were administered LEV monotherapy and 20 patients (35.1\%) who were administered LEV adjunctive therapy experienced somnolence. No significant difference in the incidence of somnolence was observed between monotherapy and adjunctive therapy groups. Regarding the incidence of somnolence in patients receiving various AEDs concomitantly, only valproic acid (VPA) showed a significantly higher incidence of somnolence compared to that in the monotherapy group.

Regarding the relationship between the daily dose of LEV and the incidence of somnolence, patients who developed somnolence received an initial LEV dose of 5-20 mg/ $\mathrm{kg}$ (median, $10 \mathrm{mg} / \mathrm{kg}$ ) and a maximum LEV dose of 5-80 mg/ $\mathrm{kg}$ (median, $20 \mathrm{mg} / \mathrm{kg}$ ). In these patients, the dose at appearance of somnolence was $5-60 \mathrm{mg} / \mathrm{kg}$ (median, $20 \mathrm{mg} / \mathrm{kg}$ )

Table 4 Distribution of levetiracetam dose in patients who developed somnolence.

\begin{tabular}{ccc}
\hline \multirow{2}{*}{ Dose of LEV $(\mathrm{mg} / \mathrm{kg})$} & \multicolumn{2}{c}{ Cases, $\mathrm{n}$} \\
\cline { 2 - 3 } & Initial dose & $\begin{array}{c}\text { Dose at appearance } \\
\text { of somnolence }\end{array}$ \\
\hline $5-10$ & 18 & 10 \\
$11-20$ & 11 & 13 \\
$21-30$ & 0 & 1 \\
$31-40$ & 0 & 2 \\
$41-50$ & 0 & 2 \\
$51-60$ & 0 & 1 \\
\hline
\end{tabular}

LEV, Levetiracetam 
Table 5 Multivariate logistic regression analysis of variables associated with somnolence

\begin{tabular}{llll}
\hline Covariate & OR & $95 \%$ CI & p-value \\
\hline Levetiracetam only & 1 & & \\
Valproic acid & 3.95 & $1.43-11.36$ & 0.0079 \\
Lamotrigine & 0.842 & $0.25-2.55$ & 0.7659 \\
Zonisamide & 0.209 & $0.01-1.22$ & 0.0878 \\
\hline
\end{tabular}

(Table 4). Of the 29 patients who developed somnolence, 19 (65.5\%) developed somnolence at the initial dose. In contrast, the patients who did not develop somnolence received an initial LEV dose of 4-20 mg/kg (median, $10 \mathrm{mg} / \mathrm{kg}$ ) and a maximum LEV dose of 5-77 mg/kg (median, $37.5 \mathrm{mg} / \mathrm{kg}$ ). Both initial dose and maximum dose were not significantly different between the groups divided by the presence or absence of somnolence ( $p=0.51$ and $p=0.67$, respectively).

In the multiple logistic regression analysis (Table 5), LEV as adjunctive therapy to VPA was identified to be a risk factor of somnolence (OR, 3.95; 95\% CI, 1.43-11.36; $\mathrm{p}=$ 0.0079) (Table 5). Lamotrigine and zonisamide were not associated with somnolence. Other AEDs such as phenobarbital, clonazepam, topiramate and carbamazepine could not be analyzed statistically due to the small numbers of patients.

\section{Discussion}

This study assessed the efficacy of LEV for seizure control and the incidence of somnolence in patients administered LEV. Both efficacy and incidence of somnolence were similar to those reported in previous studies [9-11]. The analysis of concomitantly used AEDs suggested that patients administered VPA tended to experience higher incidence of somnolence compared to those administered other AEDs.

New AEDs were developed in the early 1990 s, which led to the broadening of treatment options [12]. Conventionally, the treatment of choice for focal epilepsy was carbamazepine, and the preferred drug for general epilepsy was valproate [13]. However, in recent years, first-line AEDs have been replaced by novel AEDs [14]. Yet, despite the availability of many new AEDs with different mechanisms of action, overall outcomes for patients with newly diagnosed epilepsy have not improved [15]. For this reason, clinicians tend to choose drugs that are well tolerated with few adverse effects.

LEV is a modern AED with broadspectrum efficacy against focal and generalized epilepsy and relatively few pharmacokinetic interactions with other AEDs [1-3]. The reported efficacy of LEV in terms of responder rate is between $37 \%$ and $100 \%$ [ 9 , $10,16]$. According to these reports, treatment for idiopathic epilepsy was more effective than for epilepsy with an etiologic factor, and monotherapy was more effective than adjunctive therapy. This tendency was also observed in the findings of our study; however, the difference was not statistically significant, probably due to the small sample size.

Besides adverse effects and low tolerability, the use of LEV is associated with behav- 
ioral problems (including irritability and hyperactivity), somnolence, increased seizure frequency, and headache [9, 10]. Behavioral problems and somnolence have been reported to affect medication tolerability, although the reported frequencies vary. Egunsola et al. [4] reported $8.4 \%$ and $10.9 \%$ incidence of somnolence and behavioral problems, respectively, in children treated with LEV. Moreover, $2.2 \%$ of treated children discontinued LEV due to adverse effects. A retrospective study [9] reported that adverse effects were experienced by $17 \%$ of the patients and that the retention rate was $75 \%$ at the $6^{\text {th }}$ month and $57 \%$ at the $12^{\text {th }}$ month of LEV therapy, although the reasons for discontinuation were not given in that report. A prospective study [5] reported that $47.5 \%$ of patients experienced adverse effects, with dysphoria (31\%) being the most frequent, followed by somnolence (13\%). A phase 3 study in Japan [17] to evaluate the efficacy and safety of LEV reported that $43.8 \%$ of patients experienced somnolence. In our study, $30.5 \%$ of patients developed somnolence, resulting in a discontinuation rate of $9.4 \%$. Although previous reports have not described the dose at appearance of somnolence, this study showed that most cases $(65.5 \%)$ developed somnolence at the initial dose. The incidence of somnolence in our study and the phase 3 study in Japan were higher than those reported from other countries. This finding may suggest the involvement of a genetic factor underlying the incidence of somnolence. However, it must be noted that the method to evaluate somnolence is not well established. In previous reports mentioning somnolence [3, 9, 18], it was evaluated using only spontaneous re- ports, which do not represent an objective assessment. Although spontaneous descriptions have been used in the present study, we ensured objectivity by additionally evaluating increased sleep duration and increased frequency of daytime sleep.

Somnolence is a frequent adverse effect of almost all AEDs. To date, many reports on the incidence of somnolence with monotherapy have been reported, but to the best of our knowledge, no study has reported the development of somnolence and evaluated the associated risk factors with a combination of two or more AEDs. The results of the present study revealed that patients administered LEV as an adjunctive therapy to VPA tended to have higher incidence of somnolence compared to those administered LEV concomitantly with other AEDs, and the incidence of somnolence did not correlate with the number of concomitantly used AEDs or the daily LEV dose. Early reports found that adverse effects including somnolence were more prominent in polytherapy than in monotherapy [19]. However, recent reports have shown no significant difference between monotherapy and polytherapy in this regard [6, 7], and the results of our study support this.

Although the underlying mechanism for LEV-associated somnolence is difficult to elucidate, the following neurophysiological mechanism can be considered as a possible explanation. LEV binds to the synaptic vesicle protein SV2A, which is involved in synaptic vesicle exocytosis and presynaptic neurotransmission. Initially, LEV was thought to exhibit antiepileptic activity by inhibiting the presynaptic release of glutamate [20]. However, recent studies have reported that LEV 
acts on SV2A in an accelerated manner [21] and is involved in the release of gammaaminobutyric acid (GABA) [22]. In the context of sleep and wakefulness regulation, the ventrolateral preoptic area (POA) plays an important role in generating sleep. Chung et al. [23] reported that the GABAergic neurons in the POA project to the tuberomammillary nucleus, which is the wake-promoting center, and promote sleep. VPA has been speculated to act via multiple pathways including blockage of voltage-gated sodium channels and increase of GABA levels [24]. Hence, both VPA and LEV may act on GABAergic neurons and synergistically induce somnolence. Phenobarbital and benzodiazepines, which are strong GABA agonists, are also known to induce somnolence. However, in this study, the numbers of patients who were administered these AEDs were too small for analysis of their effect on somnolence.

The present study has a few limitations. First, since this was a retrospective study with a relatively small number of cases, LEV as adjunctive therapy to phenobarbital, clonazepam, topiramate, carbamazepine, and other AEDs could not be analyzed. Moreover, adverse effects other than somnolence could not be fully examined due to a lack of descriptive data in the medical records. Although factors such as etiology and age may affect the results, we could not examine their effects by including them as covariates. Therefore, there is a potential for certain biases. Second, several physicians treat and follow the patients with epilepsy at our hospital, and consequently the method of dosing varies. Therefore, a sufficiently objective assessment of somnolence might not have been achieved. Further prospective studies with larger sample sizes are needed to confirm the adverse effects due to the interaction between various AEDs.

In conclusion, we demonstrated that the efficacy of LEV was comparable to that reported previously, and the incidence of somnolence due to LEV administration was as high as that in previous reports. Moreover, this incidence increased when LEV was administered as adjunctive therapy to VPA. Avoiding concomitant use with VPA could decrease the rate of LEV discontinuation due to somnolence.

\section{Acknowledgements}

This study did not receive any research funding.

\section{Conflicts of interest}

The authors declare no conflicts of interest.

\section{References}

[1] Slater J, Chung S, Huynh L, Duh MS, Gorin B, McMicken C, Ziemann A, Isojarvi J. Efficacy of antiepileptic drugs in the adjunctive treatment of refractory partial-onset seizures: Meta-analysis of pivotal trials. Epilepsy Res 2018: 143: 120-129.

[2] Berkovic SF, Knowlton RC, Leroy RF, Schiemann J, Falter U. Placebocontrolled study of levetiracetam in idiopathic generalized epilepsy. Neurology 2007: 69: 1751-1760.

[3] Glauser TA, Ayala R, Elterman RD, Mitchell WG, Van Orman CB, Gauer $\mathrm{LJ}, \mathrm{Lu} \mathrm{Z}$. Double-blind placebocontrolled trial of adjunctive levetirace- 
tam in pediatric partial seizures. Neurology 2006: 66: 1654-1660.

[4] Egunsola O, Choonara I, Sammons HM. Safety of levetiracetam in paediatrics: a systematic review. PLoS One 2016: 11: e0149686.

[5] Li J, Xiao N, Chen S. Efficacy and tolerability of levetiracetam in children with epilepsy. Brain Dev 2011: 33: 145-151.

[6] Canevini MP, De Sarro G, Galimberti CA, Gatti G, Licchetta L, Malerba A, Muscas G, La Neve A, Striano P, Perucca E. Relationship between adverse effects of antiepileptic drugs, number of coprescribed drugs, and drug load in a large cohort of consecutive patients with drug-refractory epilepsy. Epilepsia 2010: 51: 797-804.

[7] Joshi R, Tripathi M, Gupta P, Gulati S, Gupta YK. Adverse effects \& drug load of antiepileptic drugs in patients with epilepsy: Monotherapy versus polytherapy. Indian J Med Res 2017: 145: 317326.

[8] Scheffer IE, Berkovic S, Capovilla G, Connolly MB, French J, Guilhoto L, Hirsch E, Jain S, Mathern GW, Moshé SL, Nordli DR, Perucca E, Tomson T, Wiebe S, Zhang YH, Zuberi SM. ILAE classification of the epilepsies: Position paper of the ILAE Commission for Classification and Terminology. Epilepsia 2017: 58: 512-521.

[9] Tekgül H, Gencpinar P, Çavuşoğlu D, Dündar. The efficacy, tolerability and safety of levetiracetam therapy in a pediatric population. Seizure 2016: 36: 1621.
[10] Weijenberg A, Brouwer OF, Callenbach PM. Levetiracetam monotherapy in children with epilepsy: a systematic review. CNS Drugs 2015: 29: 371-382.

[11] Chaisewikul R, Privitera MD, Hutton JL, Marson AG. Levetiracetam add-on for drug-resistant localization related (partial) epilepsy. Cochrane Database Syst Rev 2001: CD001901.

[12] Löscher W, Schmidt D. Modern antiepileptic drug development has failed to deliver: ways out of the current dilemma. Epilepsia 2011: 52: 657-678.

[13] Wheless JW, Clarke DF, Arzimanoglou A, Carpenter D. Treatment of pediatric epilepsy: European expert opinion, 2007. Epileptic Disord 2007: 9: 353412.

[14] Shih JJ, Whitlock JB, Chimato N, Vargas E, Karceski SC, Frank RD. Epilepsy treatment in adults and adolescents: Expert opinion, 2016. Epilepsy Behav 2017: 69: 186-222.

[15] Chen Z, Brodie MJ, Liew D, Kwan P. Treatment outcomes in patients with newly diagnosed epilepsy treated with established and new antiepileptic drugs: a 30-year longitudinal cohort study. JAMA Neurol 2018: 75: 279-286.

[16] Muramatsu K, Sawaura N, Ogata T, Makioka N, Tomita K, Motojima T, Ida K, Hazama K, Arakawa H. Efficacy and tolerability of levetiracetam for pediatric refractory epilepsy. Brain Dev 2017: 39: 231-235.

[17] Nakamura H, Osawa M, Yokoyama T, Yoshida K, Suzuki A. Efficacy and safety of levetiracetam as adjunctive therapy in Japanese children with uncontrolled 
partial-onset seizures: multicenter and open-label study (N01223), short term evaluation. Brain Nerve 2013: 65: 10831092 [Article in Japanese].

[18] Shukla G, Gupta A, Agarwal P, Poornima S. Behavioral effects and somnolence due to levetiracetam versus oxcarbazepine - a retrospective comparison study of North Indian patients with refractory epilepsy. Epilepsy Behav 2016: 64: 216-218.

[19] Perucca E. The new generation of antiepileptic drugs: advantages and disadvantages. Br J Clin Pharmacol 1996: 42: 531-543.

[20] Meehan AL, Yang X, McAdams BD, Yuan L, Rothman SM. A new mechanism for antiepileptic drug action: vesicular entry may mediate the effects of levetiracetam. J Neurophysiol 2011: 106: 1227-1239.

[21] Custer KL, Austin NS, Sullivan JM, Bajjalieh SM. Synaptic vesicle protein 2 enhances release probability at quiescent synapses. J Neurosci 2006: 26: 13031313.

[22] Tokudome K, Okumura T, Shimizu S, Mashimo T, Takizawa A, Serikawa T, Terada R, Ishihara S, Kunisawa N, Sasa M, Ohno Y. Synaptic vesicle glycoprotein 2A (SV2A) regulates kindling epileptogenesis via GABAergic neurotransmission. Sci Rep 2016: 6: 27420.

[23] Chung S, Weber F, Zhong P, Tan CL, Nguyen TN, Beier KT, Hormann N, Chang WC, Zhang Z, Do JP, Yao S, Krashes MJ, Tasic B, Cetin A, Zeng H, Knight ZA, Luo L, Dan Y. Identification of preoptic sleep neurons using retro- grade labelling and gene profiling. Nature 2017: 545: 477-481.

[24] Owens MJ, Nemeroff CB. Pharmacology of valproate. Psychopharmacol Bull 37 Suppl 2003: 2: 17-24. 